\title{
Constraint-based wave vector and frequency dependent exchange-correlation kernel of the uniform electron gas
}

\author{
Adrienn Ruzsinszky, ${ }^{1}$ Niraj K. Nepal $\odot,{ }^{1}$ J. M. Pitarke $\odot,{ }^{2,3}$ and John P. Perdew ${ }^{1,4}$ \\ ${ }^{1}$ Department of Physics, Temple University, Philadelphia, Pennsylvania 19122, USA \\ ${ }^{2}$ CIC nanoGUNE BRTA and DIPC, E-20018 Donostia, Basque Country, Spain \\ ${ }^{3}$ Materia Kondentsatuaren Fisika Saila and Centro Fisica Materiales CSIC-UPV/EHU, E-48080 Bilbao, Basque Country, Spain \\ ${ }^{4}$ Department of Chemistry, Temple University, Philadelphia, Pennsylvania 19122, USA
}

(Received 20 April 2020; accepted 27 May 2020; published 11 June 2020)

\begin{abstract}
According to time-dependent density functional theory, the exact exchange-correlation kernel $f_{x c}(n, q, \omega)$ for wave vector $q$ and frequency $\omega$ determines not only the ground-state energy but also the excited-state energies/lifetimes and time-dependent linear density response of an electron gas of uniform density $n=$ $3 /\left(4 \pi r_{s}^{3}\right)$. Here we propose a parametrization of this function based upon the satisfaction of exact constraints. For the static $(\omega=0)$ limit, we modify the model of Constantin and Pitarke to recover at small $q$ the known second-order gradient expansion, and to correct its approach to the large $q$ limit. For all $\omega$ at $q=0$, we use the model of Gross, Kohn, and Iwamoto. A Cauchy integral extends this model to complex $\omega$. Scaling relations are identified. We then combine these ingredients, damping out the $\omega$ dependence at large $q$. Away from $q=0$ and $\omega=0$, the correlation contribution to the kernel becomes dominant over exchange, even at $r_{s}=4$. The resulting correlation energies for $1 \leqslant r_{s} \leqslant 10$ from integration over imaginary $\omega$ are essentially exact. The plasmon pole of the density response function is found by analytic continuation of $f_{x c}$ to $\omega$ just below the real axis, and the resulting plasmon lifetime at $r_{s}=4$ is found for $q<k_{F}$. A static charge-density wave is found for $r_{s}>69$, and shown to be associated with softening of the plasmon mode.
\end{abstract}

DOI: 10.1103/PhysRevB.101.245135

\section{INTRODUCTION}

In time-dependent density functional theory (TDDFT) $[1-3]$, the exact linear density response function $\chi\left(\mathbf{r}, \mathbf{r}^{\prime}, \omega\right)$ of an electronic system in its ground state yields the density response $\delta n=\chi \delta v$ to a weak external scalar potential $\delta v\left(\mathbf{r}^{\prime}, \omega\right)$, oscillating at angular frequency $\omega . \chi$ provides access to the exact ground- and excited-state energies of the system. Under the standard assumption that the groundstate and time-dependent densities for the real interacting system are the same as those of a fictitious noninteracting system in an effective scalar potential (the Kohn-Sham or KS potential in the ground-state case), the true response function $\chi$ can be constructed from the calculable noninteracting response function $\chi_{\mathrm{KS}}$ and an exchange-correlation kernel $f_{x c}$. Through the adiabatic-connection fluctuation-dissipation theorem [4-6], $\chi$ yields the ground-state exchange-correlation energy from an integral along the upper half of the imaginary frequency axis. In other words, the exchange-correlation kernel $f_{x c}$ "exactifies" the random phase approximation via an effective electron-electron interaction $\frac{1}{\left|\mathbf{r}^{\prime}-\mathbf{r}\right|}+f_{x c}\left(\mathbf{r}^{\prime}, \mathbf{r}, \omega\right)$ just as ground-state Kohn-Sham density functional theory "exactifies" the Hartree approximation by addition of the exact density functional for the exchange-correlation energy. Under analytic continuation of $f_{x c}$ to complex frequencies, the poles of the response functions in the lower-half complex plane are the excitation energies/inverse lifetimes.

The exact ground-state many-electron wave function and its total energy as an expectation value of the Hamiltonian are time independent, but the electrons still move and their density still fluctuates around a time-independent average. TDDFT and the fluctuation-dissipation theorem provide a spectral decomposition of the exchange-correlation term of the total energy into contributions from correlations between density fluctuations at positive frequencies. The required integral over real frequencies would be challenging without a mathematical transformation by contour integration to the imaginary-frequency axis in the upper half of the complex plane.

For a homogeneous electronic system, Fourier transformation leads to the simple algebraic expression,

$$
\begin{gathered}
\chi(q, \omega)=\frac{\chi_{\mathrm{KS}}(q, \omega)}{\tilde{\varepsilon}(q, \omega)}, \\
\tilde{\varepsilon}(q, \omega)=1-\left[\frac{4 \pi}{q^{2}}+f_{x c}(q, \omega)\right] \chi_{\mathrm{KS}}(q, \omega),
\end{gathered}
$$

with a wave vector of magnitude $q . \chi_{\mathrm{KS}}$ is the Lindhard function [7], and the dependence of all functions upon the uniform density $n$ is implicit. For a uniform density, the exchange-correlation kernel is known to be short ranged, with a finite $q \rightarrow 0$ limit. The function $\tilde{\varepsilon}$ can vanish, introducing a collective excitation or plasmon that is not present in the noninteracting KS system.

Many exact properties of the exchange-correlation kernel have been derived, and models have been constructed to satisfy those exact constraints, in much the same way that the density functional for the ground-state exchange-correlation 
energy, $E_{x c}[n]$, is often approximated by the satisfaction of known exact constraints. Note that the uniform-gas correlation energy from quantum Monte Carlo (QMC) calculations [8], which, as extended and parametrized in Refs. [9-11], typically serves as an input to the construction of such functionals, can be accurately predicted (not fitted) by a constraint-based interpolation $[12,13]$ between known high- and low-density limits.

We know of relatively few $q$ - and $\omega$-dependent TDDFT kernels for the uniform electron gas, essentially just the Richardson-Ashcroft [14] and Constantin-Pitarke [15] kernels. Both are constructed only for imaginary (or zero) frequencies. The Richardson-Ashcroft kernel accurately predicts [16] the correlation energy per electron of the uniform electron gas, while the Constantin-Pitarke kernel has a parameter that fits that energy. The kernels commonly used in TDDFT calculations of excitation energies are usually adiabatic $(\omega=$ $0)$ and often local density approximation or LDA $(q=0)$. Our kernel is designed for the uniform electron gas, and should be used with caution for other systems. An alternative would be the tensorial kernel [17] of time-dependent current density functional theory (TDCDFT), which at the LDA level is known to be more applicable than the corresponding scalar kernel of TDDFT.

In this work, we will develop a constraint-based model kernel that refines the Constantin-Pitarke 2007 (CP07) [15] $q$-dependent static $(\omega=0)$ kernel, and combines it with the Gross-Kohn-Iwamoto [2,18] dynamic kernel for $q=0$. Our kernel is developed for general complex frequencies, while that of $\mathrm{CP} 07$ is developed only for zero and imaginary frequencies. While many practical calculations with TDDFT for real systems use the adiabatic local density approximation based upon the uniform-gas $f_{x c}(q=0, \omega=0)$, we will see that there are strong dependencies on both variables, and that, away from $q=00$ and $\omega=0$, the correlation contribution can dominate over exchange, even at the valence electron densities of metals. Our model passes several early tests: It yields very accurate correlation energies per electron for the uniform gas without fitting (while the dynamic CP07 is fitted to those energies), predicts finite lifetimes for plasmons [19] of small nonzero wave vector, and finds at about the right low density a static charge-density wave $[8,20,21]$ that arises from a softening of the plasmon mode. We did not find a chargedensity wave at any density with the original CP07 kernel. We hope that our model will have other applications, and that it might have implications for TDDFT in real systems.

The static kernel $f_{x c}(q, \omega=0)$ for the uniform electron gas (jellium) has been calculated via QMC [22] and parametrized by Corradini et al. [23]. Jellium is an important model because it has a Hamiltonian with Coulomb repulsions between electrons, but with the external potential simplified from that of positive ions to that of a uniform positive-charge background. If this background is allowed to expand or contract, then jellium is only stable for an electron density $n=3 /\left(4 \pi \mathrm{r}_{s}^{3}\right)$ with $r_{s} \approx 4$ (in atomic units or bohr), near the valenceelectron density of metallic sodium. But, by adding an appropriately chosen short-range contribution to the external potential, a stabilized jellium model can be constructed for a better description of all simple metals and their surfaces [24]. Since the bulk electron density remains uniform, all exchange- correlation effects in bulk stabilized jellium are the same as those in bulk jellium at the same density. The book by Giuliani and Vignale [25] provides a detailed discussion of exchange and correlation in the uniform electron gas, and an explanation of the important difference between the short-range kernel of the uniform electron gas [where $f_{x c}(q, \omega)$ tends to a finite constant as $q \rightarrow 0$ ] and the ultranonlocal kernels of other systems (where in this limit $f_{x c}$ diverges like $\frac{\alpha(\omega)}{q^{2}}$ ). For this and other reasons, kernel development has been generalized $[17,26,27]$ from density to current-density functionals.

Even before TDDFT, it was known that there is a local field factor $G_{x c}(q, \omega)$, with

$$
f_{x c}(q, \omega)=-\left(\frac{4 \pi}{q^{2}}\right) G_{x c}(q, \omega),
$$

that corrects the overestimation of short-range correlation in the random phase approximation (RPA) for the correlation energy of a uniform electron gas. Singwi and collaborators [28] modeled a static local field factor that essentially predicted the uniform-gas correlation energy later found from QMC [8]. Lein, Gross, and Perdew [16] used the Richardson-Ashcroft (RA) [14] dynamic kernel (as developed for imaginary frequencies only) to show that the static limit of a good kernel can capture most of the correction to the RPA correlation energy, although including the frequency dependence of the RA kernel gives even more accurate correlation energies. Here we will find that the static version of our kernel already predicts very accurate correlation energies, which are hardly changed by inclusion of our frequency dependence. But, as discussed below, the frequency dependence of the exchangecorrelation kernel is needed for other applications.

\section{DENSITY DEPENDENCE OF CONSTRAINT-BASED KERNELS}

For simplicity, we will discuss here the density dependence of constraint-based static kernels for the uniform gas. The frequency dependence complicates the notation but does not change the conclusions. Here we will use the Fermi wave vector $k_{\mathrm{F}}=\left(3 \pi^{2} n\right)^{1 / 3}$. The noninteracting response function has the simple scaling equality $\chi_{\mathrm{KS}}(q, 0)=k_{\mathrm{F}} \mathrm{F}\left(\frac{q}{2 k_{\mathrm{F}}}\right)$, and of course the Fourier transform of the Coulomb interaction between electrons is $\frac{4 \pi}{q^{2}}=k_{\mathrm{F}}^{-2} \pi\left(\frac{q}{2 k_{\mathrm{F}}}\right)^{-2}$.

The kernel has Coulomb-like scaling equalities only in the high-density and low-density limits,

$$
\begin{aligned}
f_{x c}(q, 0) & \rightarrow k_{\mathrm{F}}^{-2} G\left(\frac{q}{2 k_{\mathrm{F}}}\right), \quad\left(k_{\mathrm{F}} \rightarrow \infty\right), \\
f_{x c}(q, 0) & \rightarrow k_{\mathrm{F}}^{-2} H\left(\frac{q}{2 k_{\mathrm{F}}}\right), \quad\left(k_{\mathrm{F}} \rightarrow 0\right) .
\end{aligned}
$$

Table I shows the density dependencies of some of the ingredients of our kernel to be introduced later. The macroscopic or slowly varying-density limit is achieved when $\frac{q}{2 k_{\mathrm{F}}} \rightarrow 0$

Thus, in the high-density limit for fixed finite $\frac{q}{2 k_{\mathrm{F}}}, \tilde{\varepsilon}(q, 0) \rightarrow 1+O\left(k_{\mathrm{F}}^{-1}\right) \quad$ and $\quad \chi(q, 0) \rightarrow \chi_{\mathrm{KS}}(q, 0)$. In the adiabatic connection fluctuation dissipation expression [4-6] for the exchange-correlation energy, the exchange energy per electron $\varepsilon_{x} \sim k_{\mathrm{F}}$ arises from $\chi_{\mathrm{KS}}$ (as a function of wave vector and imaginary frequency), and the correlation 
TABLE I. Density $(n)$ dependencies of key ingredients (to be defined later) of the exchange-correlation kernel $f_{x c}(q, \omega)$ for a uniform electron gas with density parameter $r_{s}=\left(\frac{3}{4 \pi n}\right)^{1 / 3}$, and of related quantities: the Fermi wave vector $k_{\mathrm{F}}=1.9192 / r_{s}$, the bulk plasma frequency $\omega_{p}=(4 \pi n)^{1 / 2}$, and $\Delta f_{0} / f_{0}$ from Ref. [17] (atomic units).

\begin{tabular}{ccccccccc}
\hline \hline$r_{s}$ & $k_{F}$ & $\omega_{p}$ & $k^{-1 / 2} / k_{\mathrm{F}}$ & $b^{1 / 2} \omega_{p}$ & $k_{\mathrm{F}}^{2} f_{0}$ & $k_{\mathrm{F}}^{2} f_{\infty}$ & $k_{\mathrm{F}}^{2} f_{x c}(\infty, \omega)$ & $\Delta f_{0} / f_{0}$ \\
\hline 0 & $\infty$ & $\infty$ & 1.15 & $\infty$ & -3.14 & -1.89 & 0 & - \\
1 & 1.92 & 1.73 & 1.67 & 0.51 & -3.25 & -1.10 & -0.39 & -0.16 \\
2 & 0.96 & 0.61 & 1.76 & 0.49 & -3.36 & -0.92 & -0.51 & -0.12 \\
3 & 0.64 & 0.33 & 1.80 & 0.48 & -3.45 & -0.85 & -0.57 & -0.10 \\
4 & 0.48 & 0.22 & 1.82 & 0.47 & -3.53 & -0.83 & -0.61 & -0.08 \\
5 & 0.38 & 0.15 & 1.83 & 0.46 & -3.60 & -0.83 & -0.63 & -0.08 \\
$\infty$ & 0 & 0 & 2.06 & 0 & -6.07 & -3.65 & 0 & - \\
\hline \hline
\end{tabular}

energy per electron $\varepsilon_{c}$ from $\chi-\chi_{\mathrm{KS}}$ (as a function of coupling constant, wave vector, and imaginary frequency). The correlation energy is much smaller than the exchange energy at high densities, but tends to about $0.9 \varepsilon_{x}$ at very low densities. In this paper, for the ingredients of $f_{x c}$, we will employ the parametrization of Ref. [9] for the $r_{s}$ dependence of $\varepsilon_{c}$ at zero spin polarization.

\section{MODIFIED CP07 STATIC KERNEL}

We begin with the static limit of the original CP07 kernel of Eq. (12) of Ref. [16] (in atomic units):

$$
\begin{gathered}
f_{x c}^{\mathrm{CP} 07}(q, 0)=\left(\frac{4 \pi}{q^{2}}\right) B\left[e^{-k q^{2}}-1\right]-\left(\frac{4 \pi}{k_{\mathrm{F}}^{2}}\right) \frac{C}{\left[1+\frac{1}{q^{2}}\right]}, \\
k=\frac{A}{4 \pi B} .
\end{gathered}
$$

Here $A, B$, and $C$ are positive functions of density $n$ defined in Ref. [15]. These functions of density typically require derivatives of $\varepsilon_{x c}\left(r_{s}\right)$ or $\varepsilon_{c}\left(r_{s}\right)$, for which we employ the parametrizations from the Appendix of Ref. [9] (numerically almost identical to those of Refs. [10] and [11]) instead of the less accurate but simpler ones of Ref. [15]. CP07 is a constraint-based kernel that aims to reproduce the known small $q$ and large $q$ behaviors of the exact kernel:

$$
\begin{gathered}
f_{x c}(q, 0) \rightarrow-A, \quad(q \rightarrow 0), \\
f_{x c}(q, 0) \rightarrow-\left(\frac{4 \pi}{k_{\mathrm{F}}^{2}}\right) C-\left(\frac{4 \pi}{q^{2}}\right) B, \quad(q \rightarrow \infty) .
\end{gathered}
$$

Equation (8) is the well-known compressibility sum rule; approximating $f_{x c}$ by $-A$ is the adiabatic local density approximation. Equation (9) is from Refs. [22,23]. Note that $C$ arises from correlation alone, and vanishes in the high- and low-density limits, as shown in Table I.

Equation (6) was intended to recover Eq. (9), but does not because the $q \rightarrow \infty$ expansion of the last term of Eq. (6) is $-\frac{4 \pi}{k_{F}^{2}} C\left[1-\frac{1}{q^{2}}\right]$, which loses the correct $1 / q^{2}$ contribution in Eq. (9). Thus our first change to Eq. (6) is to replace $1 / q^{2}$ in the last term by $1 /\left(k q^{2}\right)^{2}$. This substitution is needed to recover Eq. (9) and the density scalings discussed in Sec. II of this article. Note that, by Table I, $k q^{2}$ scales like $\left(\frac{q}{2 k_{\mathrm{F}}}\right)^{2}$ in the high- and low-density limits.

The second change we make is to replace Eq. (8) by the more accurate and better controlled,

$$
\begin{gathered}
f_{x c}(q, 0) \rightarrow-A+D q^{2}, \quad(q \rightarrow 0), \\
D=\frac{2 C_{x c}\left(r_{s}\right)}{n^{4 / 3}},
\end{gathered}
$$

from Eqs. (25), (32), and (37) of Ref. [21], but with improved input. The $q \rightarrow 0$ limit is the limit of slowly varying-in-space induced density, in which the second-order gradient expansion becomes exact. Thus, in Eq. (11), $C_{x c}\left(r_{s}\right)$ is the coefficient of the second-order gradient expansion for the exchangecorrelation energy:

$$
C_{x c}\left(r_{s}\right)=C_{x}+C_{c}\left(r_{s}=0\right) \frac{1+3.138 r_{s}+0.3 r_{s}^{2}}{1+3.0 r_{s}+0.5334 r_{s}^{2}},
$$

with $C_{x}=-0.00238$ [29] and $C_{c}\left(r_{s}=0\right)=0.00423$ [30]. Untypically, $C_{x c}$ does not reduce to $C_{x}$ as $r_{s} \rightarrow 0$. We have used the $r_{s}$ dependence of Eq. (36) of Ref. [31], in which $C_{x c}$ decreases very slowly to zero as $r_{s}$ increases, taking the values $0.00185,0.00122$, and 0.00015 at $r_{s}=0,4$, and 70 , respectively. This means that, at very low densities with $r_{s} \geqslant$ $70, D$ will be close to 0 and the LDA kernel will be nearly correct through order $\left(\frac{q}{2 k_{\mathrm{F}}}\right)^{2}$.

A better match to the QMC kernel [22] for $r_{s}$ in the metallic range and for $\frac{q}{2 k_{\mathrm{F}}}<\sim 1$ could be achieved by setting $D=0$ in Eq. (10). Within its error bars, the QMC kernel can also be matched [32] by including higher-order terms in the gradient expansion of the exchange-correlation energy, although the fourth-order terms are not known for the correlation energy. In the interests of simplicity and generality, we have not included a $q^{4}$ term in Eq. (10). The goal of constraint satisfaction is not to match every detail, but to make a correct global map.

The result of these changes is the modified CP07 (MCP07) static kernel:

$$
\begin{aligned}
f_{x c}^{\mathrm{MCP} 07}(q, 0)= & \left(\frac{4 \pi}{q^{2}}\right) B\left[e^{-k q^{2}}\left(1+E q^{4}\right)-1\right] \\
& -\left(\frac{4 \pi}{k_{\mathrm{F}}^{2}}\right) \frac{C}{\left[1+\frac{1}{\left(k q^{2}\right)^{2}}\right]}, \\
E & =\frac{D}{4 \pi B}-\frac{k^{2}}{2} .
\end{aligned}
$$

Its exchange-only and exchange-correlation incarnations for $r_{s}=4$ are plotted in Fig. 1. We see that, away from $q=0$, correlation can be more important than exchange.

We use the name MCP07 only for the static limit of our kernel, since that is the part solely based upon a modification of the original CP07.

\section{STATIC CHARGE-DENSITY WAVE IN JELLIUM}

Overhauser [20] proposed that periodic metals could be unstable against the formation of a static charge-density wave (CDW). Quantum Monte Carlo calculations found a CDW or incipient body-centered cubic (bcc) Wigner crystallization in spin-polarized jellium at a low critical density corresponding to $r_{s}=70$ [33], or at $r_{s}=85 \pm 20$ in spin-unpolarized jellium 


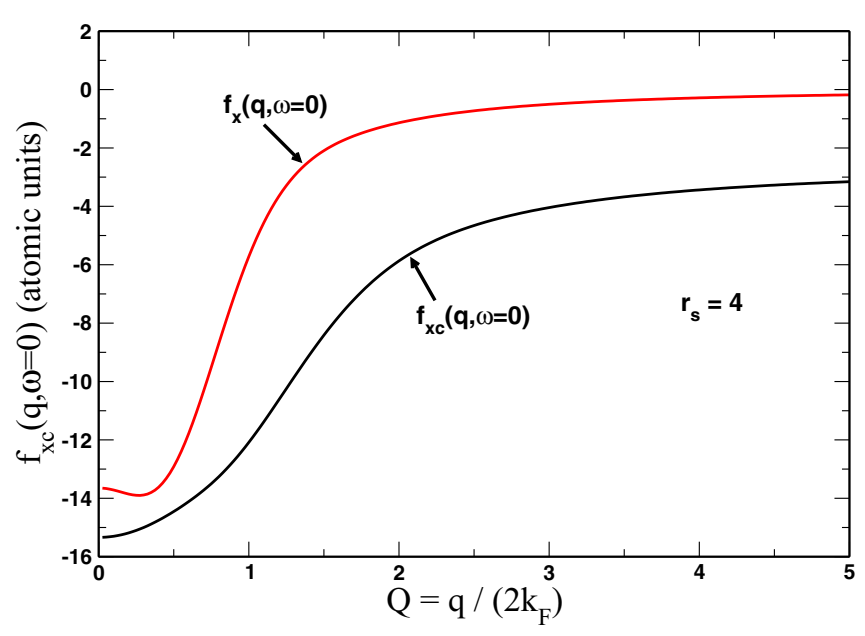

FIG. 1. Modified CP07 (MCP07) static kernels for jellium with density parameter $r_{s}=4$ at the exchange-only and exchangecorrelation levels, versus reduced wave vector.

[8]. The 1980 calculation of Ceperley and Alder [8] also found that ground-state jellium remains spin unpolarized for $r_{s} \leqslant 75 \pm 5$. In the same year, Perdew and Datta [21], using a static kernel designed to satisfy Eqs. (10) and (11), also found a CDW near this critical $\mathrm{r}_{s}$.

Figure 2 of the present article, which is similar to Fig. 4 of Ref. [21], was found by fixing a value for $\frac{q}{2 k_{\mathrm{F}}}$ and then searching for the largest value of $k_{\mathrm{F}}$ that makes $\tilde{\epsilon}(q)=0$ (hence a nonzero density response at wave vector $q$ even in the absence of any perturbing potential). This happens around $r_{s}=30$ in the adiabatic local density approximation, and around $r_{s}=69$ in MCP07. We could not find a charge-density wave at any density from the original CP07. All the lowdensity instabilities of jellium are difficult to pinpoint, because the energies of the different phases as functions of $r_{s}$ are nearly the same at low densities.

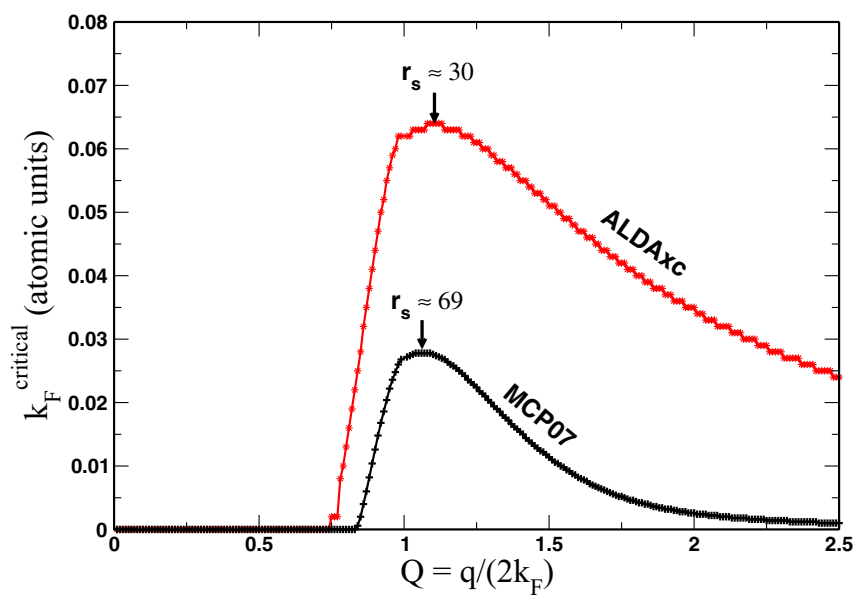

FIG. 2. Critical Fermi wave vector for the appearance of a static charge-density wave in a low-density jellium, from the adiabatic local density approximation $\left[f_{x c}=f_{x c}(0,0)\right]$ and MCP07 static $\left[f_{x c}=f_{x c}(q, 0)\right]$ exchange-correlation kernels, versus reduced wave vector.
The charge-density wave first appears with $\frac{q}{2 k_{\mathrm{F}}} \approx 1.14$, making $q$ close to the first reciprocal lattice vector of a bcc Wigner crystal with one electron per primitive cell. For much smaller $\frac{q}{2 k_{\mathrm{F}}}$, the CDW is strongly suppressed by the Coulomb term $4 \pi / q^{2}$ in Eq. (2). Later in this article, we will show that the CDW is associated with a softening of the plasmon mode.

Our Fig. 1 shows that the static MCP07 kernel $f_{x c}(q, 0)$ is always more negative than its exchange-only version $f_{x}(q, 0)$. This result confirms Overhauser's 1968 prediction that correlation enhances the charge-density wave [20].

\section{FREQUENCY-DEPENDENT LOCAL KERNEL OF GROSS AND KOHN}

A constraint-based model for $f_{x c}(q=0, \omega)$ was proposed in 1985 by Gross and Kohn [2], and later corrected by Iwamoto and Gross [18]. It starts from a constrained interpolation for the imaginary part, evaluated at a real frequency, between known real zero- $\left(f_{0}\right)$ and infinite- $\left(f_{\infty}\right)$ frequency limits at $q=0$ :

$$
\begin{gathered}
\operatorname{Im} f_{x c}(0, \omega)=-c b^{3 / 4} g\left(b^{1 / 2} \omega\right), \\
g(x)=\frac{x}{\left(1+x^{2}\right)^{5 / 4}}, \\
b=\left\{\left(\frac{\gamma}{c}\right)\left[f_{\infty}-f_{0}\right]\right\}^{4 / 3}, \\
c=23 \frac{\pi}{15}, \\
\gamma=\frac{\left[\Gamma\left(\frac{1}{4}\right)\right]^{2}}{(32 \pi)^{1 / 2}}=1.311 .
\end{gathered}
$$

Figure 3 shows this function of real $\omega$ for $r_{s}=4$, and also its exchange-only contribution. Again the importance of correlation is manifest. Table I shows that the dimensionless quantity $b^{1 / 2} \omega_{p}$ (where $\omega_{p}=(4 \pi n)^{1 / 2}$ is the bulk plasmon frequency) is nearly constant over the range of metallic densities, but not over all densities. Thus, in the metallic range, $g\left(b^{1 / 2} \omega\right)$ is approximately a function of $\frac{\omega}{\omega_{p}}$.

The next step is to use the Kramers-Kronig relations [2] between the imaginary and real parts of $f_{x c}-f_{\infty}$ at real frequency to find

$$
\operatorname{Re} f_{x c}(0, \omega)-f_{\infty}=\left(\frac{1}{\pi}\right) P \int_{-\infty}^{\infty} d \omega^{\prime} \frac{\operatorname{Im} f_{x c}\left(0, \omega^{\prime}\right)}{\omega^{\prime}-\omega} .
$$

As $\omega \rightarrow \infty, \quad \operatorname{Im} f_{x c}(0, \omega) \sim-c / \omega^{3 / 2}$ and $\operatorname{Re} f_{x c}(0, \omega)-$ $f_{\infty} \sim c / \omega^{3 / 2}$.

The principal value of the integral can be found numerically. However, the scaling relation of Eq. (15) implies the scaling relation,

$$
\operatorname{Re} f_{x c}(0, \omega)-f_{\infty}=-c b^{3 / 4} h\left(b^{1 / 2} \omega\right),
$$

where $h(0)=\frac{1}{\gamma}$ to recover the correct nonzero $\omega \rightarrow 0$ limit. A fair fit with the correct large- $\omega$ asymptotics is provided by the simple algebraic model,

$$
h_{\text {model }}(x)=\frac{\left(\frac{1}{\gamma}\right)\left[1-a x^{2}\right]}{\left[1+(a / \gamma)^{4 / 7} x^{2}\right]^{7 / 4}},
$$

with a fit parameter $a=0.63$. 


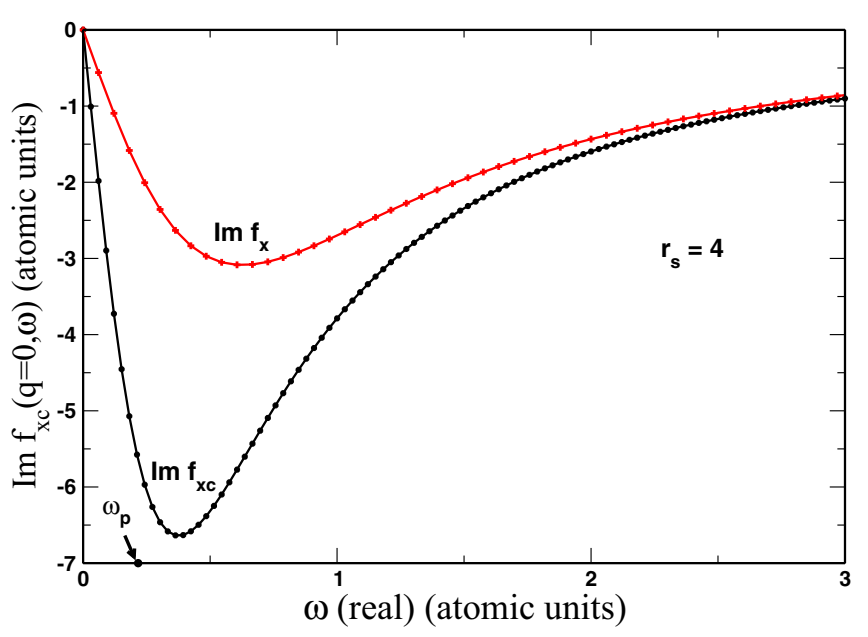

FIG. 3. Imaginary part of the Gross-Kohn $q=0$ dynamic kernel for jellium with density parameter $r_{s}=4$, at the exchange-only and exchange-correlation levels, versus real frequency.

Figure 4 compares the real-frequency dependencies of the real part of the kernel, with and without correlation, from the Kramers-Kronig relation and from the model. The model is less accurate at intermediate frequencies than at low or high frequencies. While the Kramers-Kronig choice is the consistent one, we have found that it does not make any significant difference from the model in the applications presented here.

For calculation of the correlation energy, we will need $f_{x c}(0, \omega)$ for frequencies $\omega$ in the upper-half complex plane, where this function is analytic [2]. For this, we use the Cauchy integral over real $\omega^{\prime}$ :

$$
f_{x c}(0, \omega)-f_{\infty}=\frac{1}{2 \pi i} \int_{\infty}^{\infty} d \omega^{\prime} \frac{\left[f_{x c}\left(0, \omega^{\prime}\right)-f_{\infty}\right]}{\omega^{\prime}-\omega},
$$

which follows from the residue theorem and exact properties of $f_{x c}(0, \omega)-f_{\infty}$ [2]. By letting $\omega$ approach the real axis from above, we can derive the Kramers-Kronig relations including

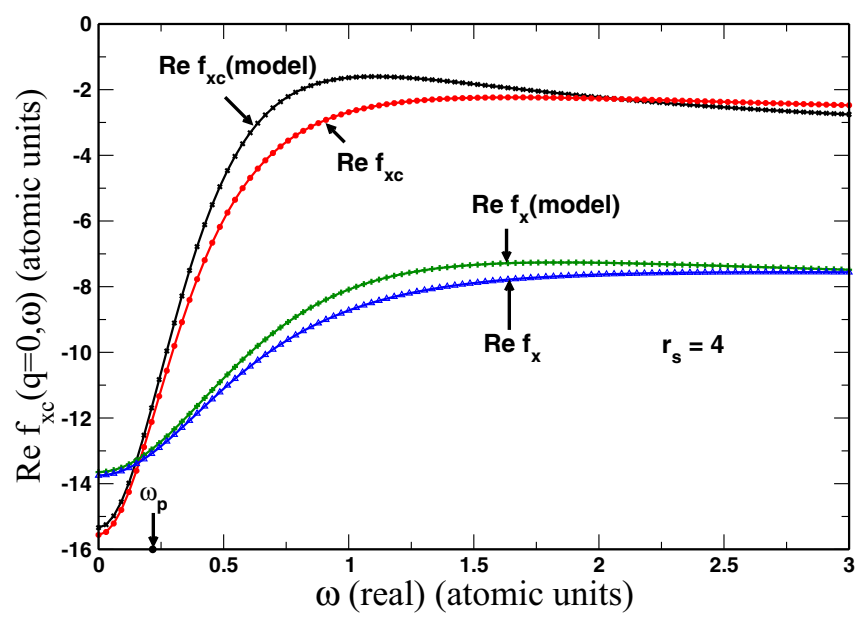

FIG. 4. Real part of the Gross-Kohn $q=0$ dynamic kernel for jellium with density parameter $r_{s}=4$, at the exchange-only and exchange-correlation levels, versus real frequency [from the Kramers-Kronig relation of Eq. (20) and from the model of Eq. (22)].

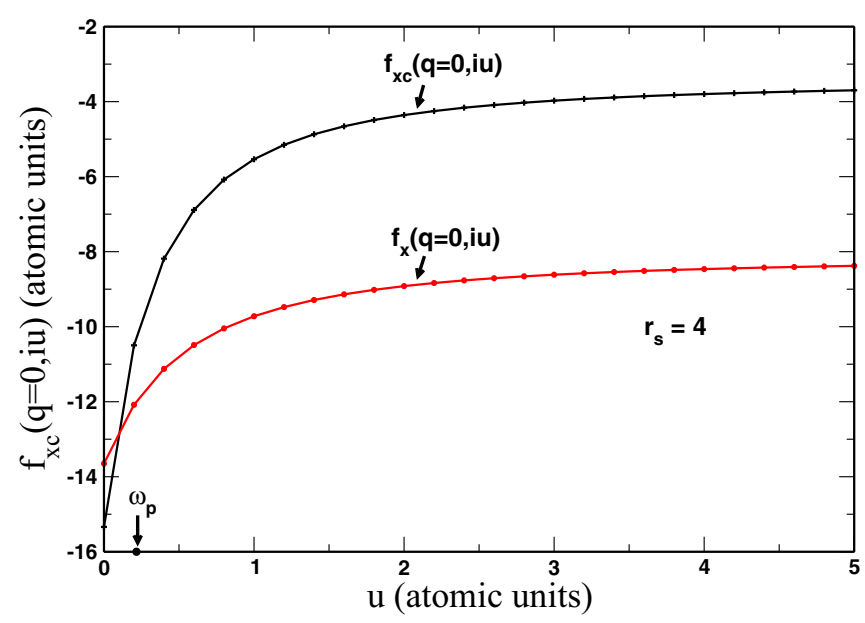

FIG. 5. The purely real Gross-Kohn $q=0$ dynamic kernel for jellium with density parameter $r_{s}=4$, at the exchange-only and exchange-correlation levels, versus imaginary frequency [from the Cauchy integral of Eq. (23) and the model of Eq. (22)].

Eq. (20). Figure 5 shows the kernel for frequencies on the upper imaginary axis, where the kernel is purely real.

\section{COMBINING THE WAVE-VECTOR DEPENDENCE OF MCP07 WITH THE FREQUENCY DEPENDENCE OF THE GROSS-KOHN KERNEL}

An important constraint is Eq. (5.176) of Ref. [25], attributed there to Ref. [34]. It says that the $\omega$ dependence of the kernel damps out at large $q$, even when the kernel itself has a nonzero large- $q$ limit.

To avoid empiricism, we will use the same Gaussian damping factor that damps out the local density and gradient expansion terms at large $q$ in Eq. (13):

$$
f_{x c}(q, \omega)=\left[1+e^{-k q^{2}}\left\{\frac{f_{x c}(0, \omega)}{f_{x c}(0,0)}-1\right\}\right] f_{x c}^{\mathrm{MCP} 07}(q, 0) \text {. }
$$

When $q=0$, Eq. (24) properly recovers $f_{x c}(0, \omega)$. When $\omega=$ 0 , Eq. (24) properly recovers $f_{x c}^{\mathrm{MCP} 07}(q, 0)$. And when $q \rightarrow$ $\infty$, Eq. (24) correctly reduces to $f_{x c}^{\mathrm{MCP} 07}(q, 0)$.

Figure 6 shows the $q$ dependence of the imaginary part of Eq. (24) for $r_{s}=4$ for various real frequencies that are integer multiples of the bulk plasmon frequency. Figure 7 shows the same for the real part [using the model of Eq. (22)]. Note that the frequency dependence is already strongly damped at $\frac{q}{2 k_{\mathrm{F}}}=1$.

A viscosity correction to the compressibility value for $f_{0}=$ $f_{x c}(0,0)$ was found by Conti and Vignale [35]. It is of order $10 \%$ at metallic densities, as shown by the values of $\Delta f_{0} / f_{0}$ in Table I (based on $\Delta f_{0}$ values from Ref. [17]), and is not included in our Eq. (24).

\section{PLASMON IN JELLIUM}

The plasmon is a collective long-wavelength oscillation of the electron density, at a frequency $\omega_{p}(q)$ that tends as $q \rightarrow 0$ to the classical limit or bulk plasmon frequency $\omega_{p}=$ $(4 \pi n)^{1 / 2}$. At $q$ less than a critical wave vector $q_{c}$, the real part of the complex plasmon energy $\omega_{p}(q)$ lies above the highest 


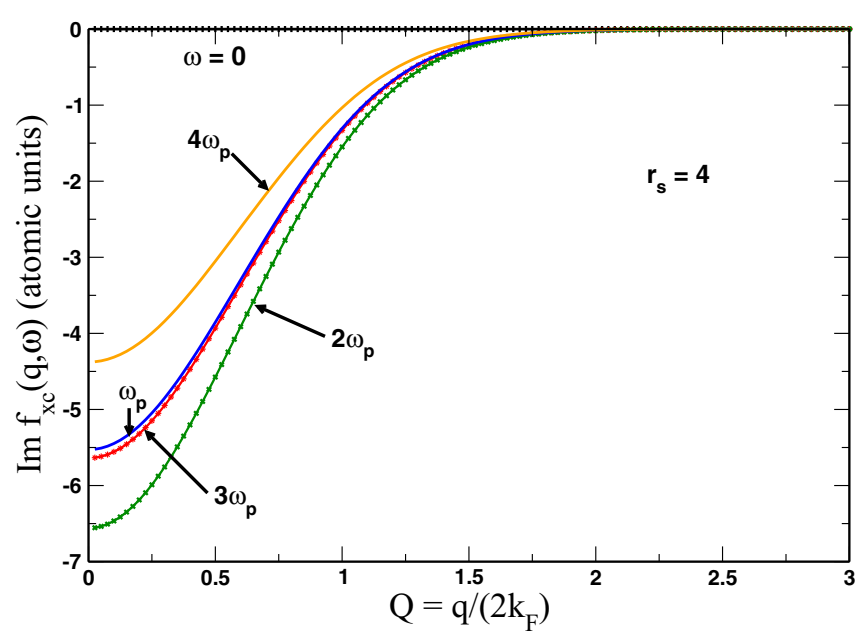

FIG. 6. Imaginary part of the exchange-correlation kernel of Eq. (24) for jellium with density parameter $r_{s}=4$, for five different real frequencies, versus reduced wave vector.

energy of the continuum of single electron-hole excitations of wave vector $q$, which in a noninteracting picture has a highest energy of $\frac{\left(k_{\mathrm{F}}+q\right)^{2}}{2}-\frac{k_{\mathrm{F}}^{2}}{2}$. Thus

$$
\frac{\operatorname{Re} \omega_{p}\left(q_{c}\right)}{k_{\mathrm{F}}^{2}}=\left(\frac{1}{2}\right)\left(\frac{q_{c}}{k_{\mathrm{F}}}\right)^{2}+\frac{q_{c}}{k_{\mathrm{F}}} .
$$

In the range $q<q_{c}$ (the only range we will consider here), the plasmon excitation cannot decay to a single electron-hole pair excitation, so its lifetime is infinite for any real (hence frequency-independent kernel). But a frequency-dependent kernel should yield a plasmon frequency in the lower-half complex frequency plane, where $\operatorname{Im} \omega_{p}(q)$ is minus the inverse of a lifetime arising from decay of the plasmon into multiple electron-hole pairs.

We find $\omega_{p}(q)$ by fixing a real wave vector $q$ and searching over complex frequencies $\omega$ for the one that zeros out $\tilde{\varepsilon}(q, \omega)$ of Eq. (2). In practice, we stop when $|\tilde{\varepsilon}|$ is of order $10^{-3}$. Since

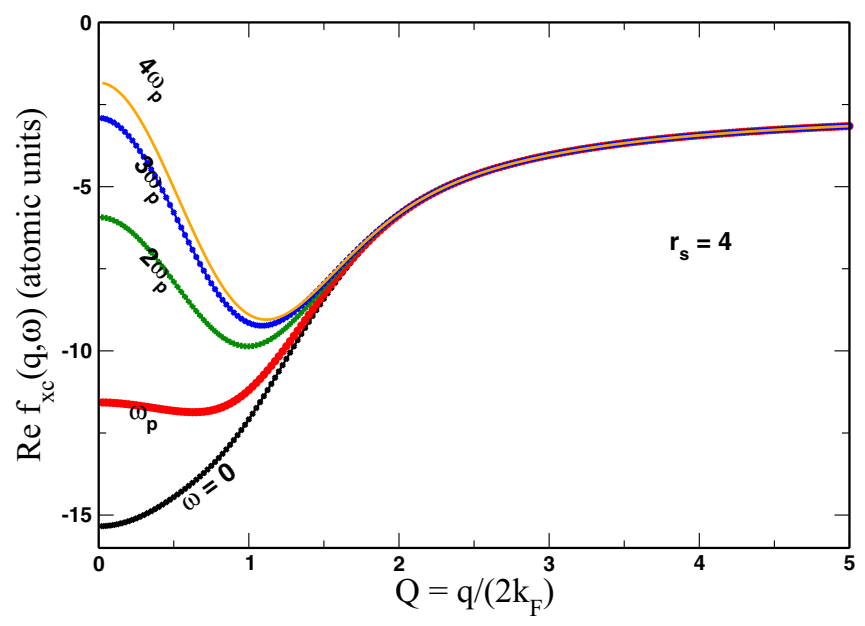

FIG. 7. Real part of the exchange-correlation kernel of Eq. (24) for jellium with density parameter $r_{s}=4$, for five different real frequencies, versus reduced wave vector [from the model of Eq. (22)].

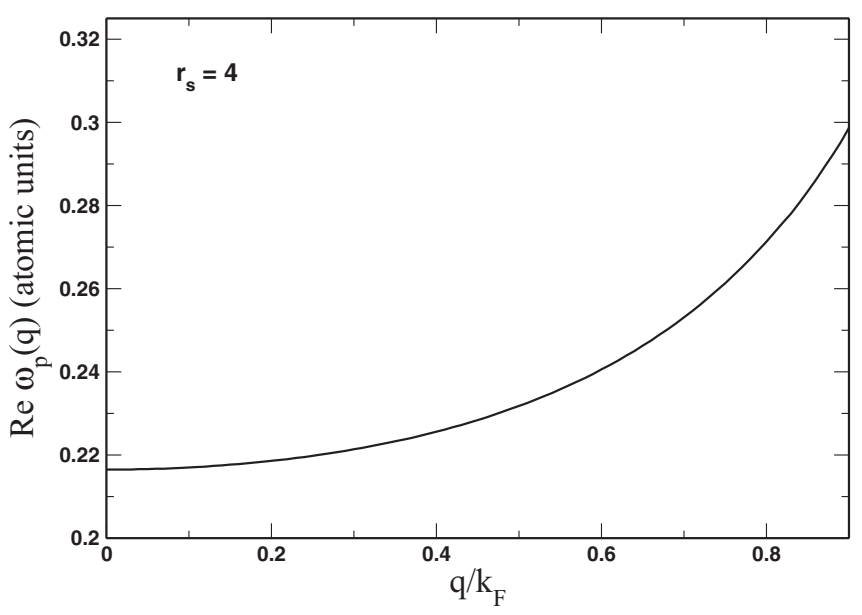

FIG. 8. Plasmon dispersion for jellium with density parameter $r_{s}=4$, from the kernel of Eq. (24), versus reduced wave vector. The real part of the plasmon frequency is plotted.

our Cauchy integral of Eq. (23) is only for $\omega$ in the upperhalf complex plane, we find $f_{x c}(0, \omega)$ by analytic continuation or Taylor expansion from a near frequency on the real axis. The zeroth-order term of this expansion almost suffices, as we confirm by adding the first-order term, using the CauchyRiemann conditions on an analytic function to convert known derivatives of the real and imaginary parts of $f_{x c}$ with respect to $\operatorname{Re} \omega$ to derivatives with respect to $\operatorname{Im} \omega$.

Figure 8 shows the resulting plasmon dispersion or $\mathrm{Re}$ $\omega_{p}(q)$ for $r_{s}=4$, which would be almost the same if the frequency dependence of the kernel were neglected, and not qualitatively different if the kernel were set to 0 . Figure 9 shows the resulting $\operatorname{Im} \omega_{p}(q)$, or minus the inverse plasmon lifetime, which would equal zero without the frequency dependence. The calculated inverse lifetime grows like $q^{2}$ at small $q$, as expected [25,36], but starts to decrease again as $q$ approaches $k_{\mathrm{F}}$, where the Gross-Kohn frequency dependence is increasingly damped out via our Eq. (24). The minimum predicted plasmon lifetime is of the order of femtoseconds.

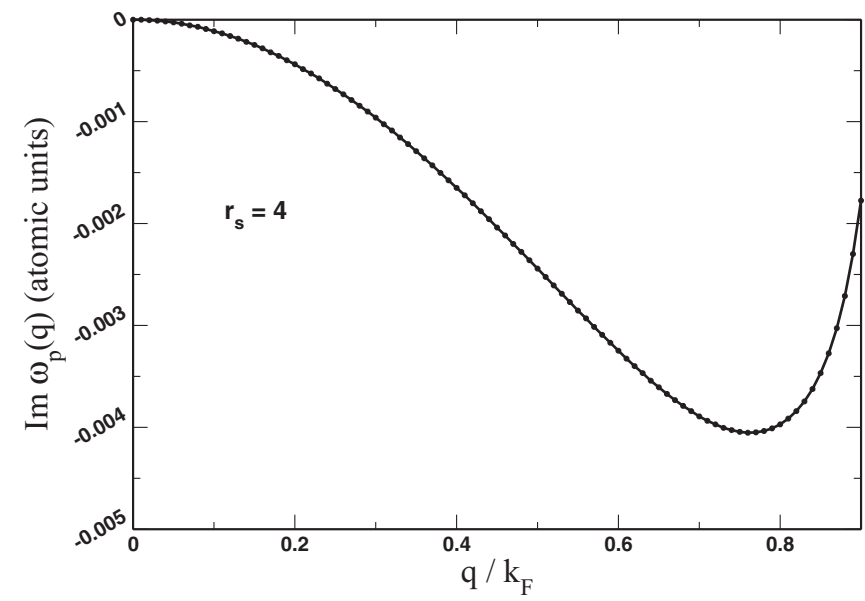

FIG. 9. Plasmon damping for jellium with density parameter $r_{s}=4$, from the kernel of Eq. (24), versus reduced wave vector. The imaginary part of the plasmon frequency is plotted. 


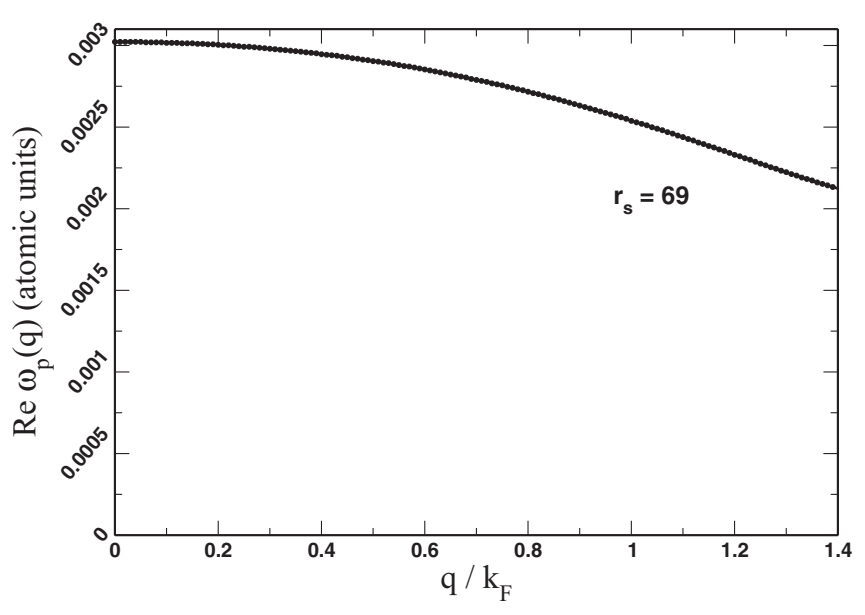

FIG. 10. Plasmon dispersion for jellium with density parameter $r_{s}=69$, from the kernel of Eq. (24), versus reduced wave vector. The softened plasmon mode may lead to the static charge-density wave.

Figure 10 shows $\operatorname{Re} \omega_{p}(q)$ for $r_{s}=69$, where the static charge density wave was found to appear in Sec. IV. Unlike the dispersion in Fig. 8, the dispersion here is downward, and $\omega_{p}(q)$ appears to be heading toward zero at $q / k_{\mathrm{F}} \approx 2$. Thus the static charge density wave can be understood to arise from a soft plasmon mode.

\section{CORRELATION ENERGY PER ELECTRON IN JELLIUM}

The correlation energy of the uniform electron gas has a long history, going back to the random phase approximation (RPA) $\left(f_{x c}=0\right)$ of the 1950s [4]. The formula we use here is Eq. (27) of Ref. [16]. In this equation, an integral over real frequencies from 0 to $\infty$ has been transformed by contour integration to an integral over imaginary frequencies in the upper half plane. This is done to avoid the plasmon pole near the real axis, and results in the smooth frequency integrand shown in Figs. 3 and 4 of Ref. [16]. The Kohn-Sham noninteracting and real interacting systems are connected adiabatically through the coupling constant between 0 and 1 in the Coulomb interaction $\frac{4 \pi \lambda}{q^{2}}$. The exchange-correlation kernel must also be scaled, as in Eq. (18) of Ref. [16]:

$$
f_{x c}^{\lambda}(n, q, \omega)=\lambda^{-1} f_{x c}\left(\frac{n}{\lambda^{3}}, \frac{q}{\lambda}, \frac{\omega}{\lambda^{2}}\right) .
$$

Figure 11 shows our results for the correlation energy per electron as a function of $r_{s}$ in the metallic range, in comparison with the highly accurate parametrization and extension [13] of the results of Ref. [8] by Perdew and Wang 1992 [11] (indistinguishable on the scale of the figure from the parametrization of Ref. [9]). As is well known, $\operatorname{RPA}\left(f_{x c} \rightarrow 0\right)$ makes the correlation energy per electron too low by about 0.4 $\mathrm{eV} /$ electron, and the adiabatic local density approximation $\left[f_{x c} \rightarrow f_{x c}(0,0)\right]$ makes it too high by about the same absolute error. A good kernel $f_{x c}(q, \omega)$ should produce an accurate result, and our static MCP07 kernel does so to a remarkable extent. Adding the frequency dependence of Eq. (24) degrades the accuracy, but almost negligibly. Replacing the

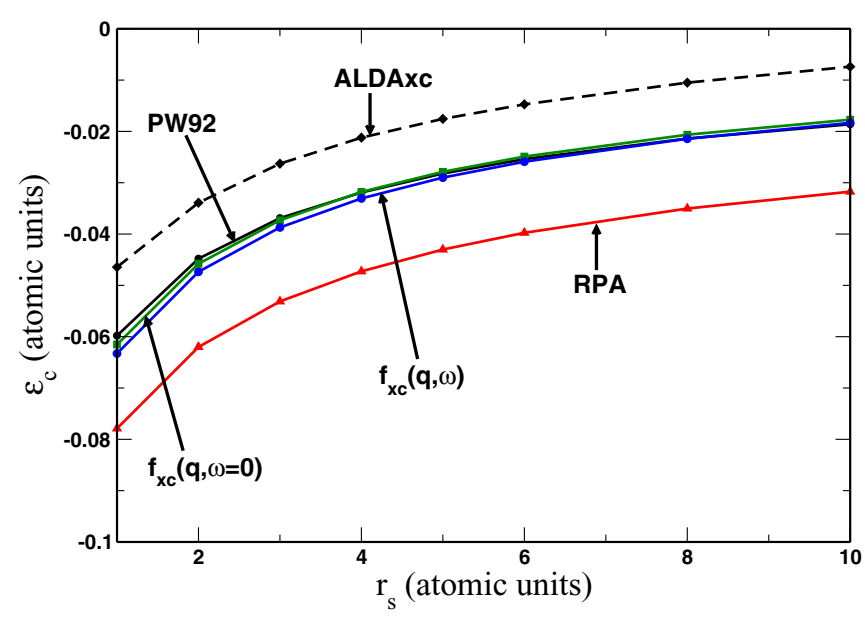

FIG. 11. Correlation energy per electron for jellium from various kernels, and the essentially exact Perdew-Wang 1992 (PW92) parametrization, versus density parameter $r_{s}$. The RPA has no kernel $\left(f_{x c}=0\right)$. The adiabatic local density approximation $\left[f_{x c}=\right.$ $\left.f_{x c}(0,0)\right]$, the static MCP07 kernel of Eq. (13) $\left[f_{x c}=f_{x c}(q, 0)\right]$, and the full dynamic kernel of Eq. (24) $\left[f_{x c}=f_{x c}(q, \omega)\right]$ are also tested here. The wave-vector dependence and frequency dependence make the kernel $f_{x c}(q, \omega)$ less negative (Figs. 1 and 5), which moves the kernel-corrected correlation energy closer to RPA, in which the kernel is zero.

Gaussian in Eq. (24) by 1 (thus using the undamped GrossKohn frequency dependence) would degrade the accuracy significantly, correcting only about two-thirds of the RPA error.

In both this work and Ref. [16], the frequency dependence of the exchange-correlation kernel has very little effect on the correlation energy of the uniform electron gas. But the frequency dependence is what produced the plasmon inverse lifetime in Fig. 9. Other properties that are or may be sensitive to the frequency dependence are listed in the Conclusions section.

\section{CONCLUSIONS}

The CP07 exchange-correlation kernel for zero frequency and the Gross-Kohn kernel for zero wave vector were constructed for the uniform electron gas via the satisfaction of exact constraints. By imposing further exact constraints, we have made an improved MCP07 static kernel and combined it with the Gross-Kohn dynamic kernel. Key added constraints include the second-order gradient expansion for the exchangecorrelation energy, and the damping out of the frequency dependence with increasing wave vector. That damping out is already substantial at $q \approx k_{\mathrm{F}}$.

Without any fitting, we have achieved high accuracy for all studied properties. In particular, the critical density $\left(r_{s} \approx 69\right)$ and critical wave vector of the static charge-density wave that appears at low density are accurate. We have shown that this ground-state instability of the uniform phase is associated with a soft plasmon. We have also found that correlation enhances the instability, as Overhauser predicted in 1968 [20]. 
We have also studied the plasmon at the density of metallic sodium $\left(r_{s}=4\right)$, where our frequency dependence produces a plasmon lifetime that first decreases from infinity to a few femtoseconds and then increases, as the wave vector increases from 0 toward the Fermi wave vector $k_{\mathrm{F}}$. Previous work [25,36], based on a Taylor expansion for $q \ll k_{F}$, found a monotonic decrease in lifetime with increasing $q$ in the latter range.

We have also calculated remarkably accurate correlation energies per electron for metallic $r_{s}$ from 1 to 10 . The improvement over RPA arises from the wave-vector dependence of the MCP07 kernel. The frequency dependence of our kernel has almost no effect on the ground-state energy, a conclusion that might extend to real systems.

It should be noted that [37], for metallic densities, the range of wave vectors relevant to the plasmon outside the electronhole continuum, $0 \leqslant q<\sim k_{\mathrm{F}}$, is different from the range relevant to the correlation energy, $0<\sim q<\sim 3 k_{\mathrm{F}}$. At much lower densities, the latter range is also relevant to the plasmon and charge-density wave. In the range $0 \leqslant q<\sim k_{\mathrm{F}}$, the effective interaction $\frac{4 \pi}{q^{2}}+f_{x c}$ in Eq. (2) is dominated by $\frac{4 \pi}{q^{2}}$ [37], so modest deviations of $f_{x c}$ from its RPA value 0 , or better from the adiabatic local density approximation $f_{x c}(0,0)$, have almost negligible effect in that range, apart from emergent phenomena like the plasmon lifetime. Thus our applications test the kernel not only over a wide range of densities but also over a fairly wide range of $\frac{q}{2 k_{\mathrm{F}}}$. In the future, we hope to find more demanding tests for the frequency dependence. Several properties of uniform or weakly inhomogeneous densities are expected to be, unlike the correlation energy of the uniform gas, sensitive to the frequency dependence of the uniform gas kernel, including the electronic stopping power [38] and residual resistivity [39], optical absorption of a weakly inhomogeneous electron density [40], and the peak widths of the wave-vector-dependent dynamical structure factor [41].

In our uniform-gas exchange-correlation kernel, the full Gross-Kohn frequency dependence is unveiled only in the long-wavelength $(q \rightarrow 0)$ limit, in which the kernel itself is overwhelmed by the Coulomb interaction $\frac{4 \pi}{q^{2}}$. Inhomogeneous ground states [25] have ultranonlocal kernels with an $\frac{\alpha(\omega)}{q^{2}}$ variation in this limit that strongly affects optical absorption, and might quantitatively correct the qualitatively right RPA description of long-range van der Waals interaction.

\section{ACKNOWLEDGMENTS}

A.R. and N.K.N. acknowledge support from the U.S. National Science Foundation under Grant No. DMR-1553022. J.P.P. acknowledges support from the U.S. National Science Foundation under Grant No. DMR-1939528 (CMMT-Division of Materials Theory, with a contribution from CTMC-Division of Chemistry).
[1] E. Runge and E. K. U. Gross, Phys. Rev. Lett. 52, 997 (1984).

[2] E. K. U. Gross and W. Kohn, Phys. Rev. Lett. 55, 2850 (1985); 57, 923 (1986).

[3] M. Petersilka, U. J. Gossmann, and E. K. U. Gross, Phys. Rev. Lett. 76, 1212 (1996).

[4] P. Nozières and D. Pines, Nuovo Cimento 9, 470 (1958).

[5] D. C. Langreth and J. P. Perdew, Solid State Commun. 17, 1425 (1975).

[6] O. Gunnarsson and B. I. Lundqvist, Phys. Rev. B 13, 4274 (1976).

[7] J. Lindhard, Dan. Mat. Fys. Medd. 28, 1 (1954).

[8] D. M. Ceperley and B. J. Alder, Phys. Rev. Lett. 45, 566 (1980).

[9] J. P. Perdew and A. Zunger, Phys. Rev. B 23, 5048 (1981).

[10] S. H. Vosko, L. Wilk, and M. Nusair, Can. J. Phys. 58, 1200 (1980).

[11] J. P. Perdew and Y. Wang, Phys. Rev. B 45, 13244 (1992).

[12] J. Sun, J. P. Perdew, and M. Seidl, Phys. Rev. B 81, 085123 (2010).

[13] P. Bhattarai, A. Patra, C. Shahi, and J. P. Perdew, Phys. Rev. B 97, 195128 (2018).

[14] C. F. Richardson and N. W. Ashcroft, Phys. Rev. B 50, 8170 (1994).

[15] L. A. Constantin and J. M. Pitarke, Phys. Rev. B 75, 245127 (2007).

[16] M. Lein, E. K. U. Gross, and J. P. Perdew, Phys. Rev. B 61, 13431 (2000)

[17] Z. Qian and G. Vignale, Phys. Rev. B 65, 235121 (2002).

[18] N. Iwamoto and E. K. U. Gross, Phys. Rev. B 35, 3003 (1987).
[19] K. Tatarczyk, A. Schindlmayr, and M. Scheffler, Phys. Rev. B 63, 235106 (2001).

[20] A. W. Overhauser, Phys. Rev. 167, 691 (1968).

[21] J. P. Perdew and T. Datta, Phys. Stat. Sol. (b) 102, 283 (1980).

[22] S. Moroni, D. M. Ceperley, and G. Senatore, Phys. Rev. Lett. 75, 689 (1995).

[23] M. Corradini, R. Del Sole, G. Onida, and M. Palummo, Phys. Rev. B 57, 14569 (1998).

[24] J. P. Perdew, H. Q. Tran, and E. D. Smith, Phys. Rev. B 42, 11627 (1990)

[25] G. F. Giuliani and G. Vignale, Quantum Theory of the Electron Liquid (Cambridge University Press, Cambridge, 2005).

[26] G. Vignale and W. Kohn, Phys. Rev. Lett. 77, 2037 (1996).

[27] C. A. Ullrich and K. Burke, J. Chem. Phys. 121, 28 (2004).

[28] K. S. Singwi, A. Sjölander, M. P. Tosi, and R. H. Land, Phys. Rev. B 1, 1044 (1970).

[29] P. R. Antoniewicz and L. Kleinman, Phys. Rev. B 31, 6779 (1985).

[30] S. K. Ma and K. A. Brueckner, Phys. Rev. 165, 18 (1968).

[31] A. Cancio, G. P. Chen, B. T. Krull, and K. Burke, J. Chem. Phys. 149, 084116 (2018).

[32] J. Tao, J. P. Perdew, L. M. Almeida, C. Fiolhais, and S. Kümmel, Phys. Rev. B 77, 245107 (2008).

[33] D. Ceperley, Phys. Rev. B 18, 3126 (1978).

[34] A. Holas, in Strongly Coupled Plasma Physics, edited by F. J. Rogers and H. Dewitt, NATO Advanced Study Institute Series B: Vol. 154 (Plenum, New York, 1986), pp. 463-482.

[35] S. Conti and G. Vignale, Phys. Rev. B 60, 7966 (1999). 
[36] G. Vignale, C. A. Ullrich, and S. Conti, Phys. Rev. Lett. 79, 4878 (1997).

[37] N. K. Nepal, S. Adhikari, B. Neupane, S. Ruan, S. Neupane, and A. Ruzsinszky, Phys. Rev. B 101, 195137 (2020).

[38] V. U. Nazarov, J. M. Pitarke, Y. Takada, G. Vignale, and Y.-C. Chang, Phys. Rev. B 76, 205103 (2007).
[39] V. U. Nazarov, G. Vignale, and Y.-C. Chang, Phys. Rev. B 89, 241108(R) (2014).

[40] V. U. Nazarov, G. Vignale, and Y.-C. Chang, Phys. Rev. Lett. 102, 113001 (2009).

[41] B. C. Larson, J. Z. Tischler, E. D. Isaacs, P. Zschack, A. Fleszar, and A. G. Eguiluz, Phys. Rev. Lett. 77, 1346 (1996). 